
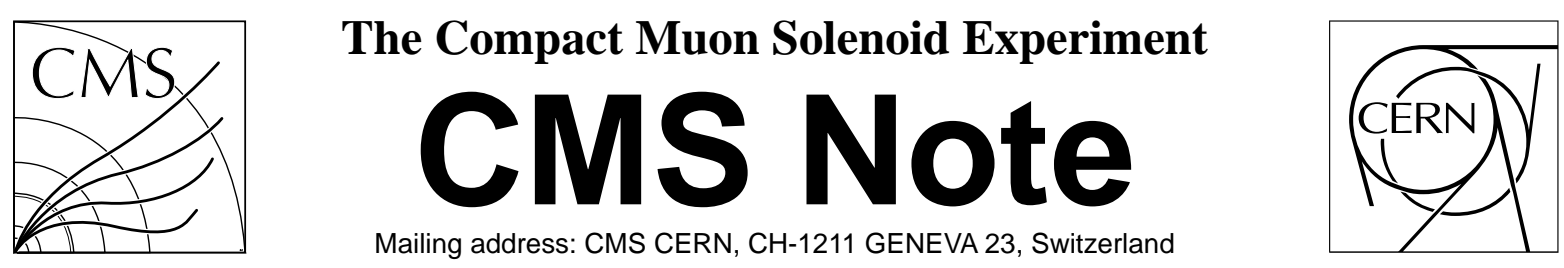

31 July 1997

\title{
Explicit Covariance Matrix for Particle Measurement Precision
}

\author{
V. Karimäki \\ Helsinki Institute of Physics, Helsinki, Finland
}

\begin{abstract}
We derive explicit and precise formulae for 3 by 3 error matrix of particle transverse momentum, direction and impact parameter. The error matrix elements are expressed as functions of up to fourth order statistical moments of the measured coordinates. The formulae are valid for any curvature and track length in case of negligible multiple scattering. The calculation is compared with formulae derived by Gluckstern for curvature and direction. We show that Gluckstern formulation is valid at the limit of small $L / R$, ratio between the track length and radius of curvature.
\end{abstract}




\section{Introduction}

Search for massive new particles such as Higgs boson in high energy experiments involves measurement of high transverse momentum particles. Therefore an important feature of the detector is its capability to measure high $p_{t}$ trajectory parameters with a good precision. In designing new detectors one normally has to use sophisticated program codes in order to understand the resolution requirements of a heterogeneous detector system often consisting of several detector types with variable resolutions. Explicit precision formulae easy to use are therefore of great interest. In this paper we derive new formulae for this purpose.

Earlier works exist on explicit calculation of particle measurement precision. Amongst the most frequently cited is Gluckstern's evaluation of uncertainties in particle momentum and direction due to multiple scattering and measurement errors [1]. Gluckstern uses parabola description of the projected trajectory. In this early work no impact parameter uncertainties were considered. Since the discovery of higher flavour particles the impact parameter analysis has become an important method to enhance the experimental event samples with short living flavour particles. In a more recent work Innes [2] uses the Gluckstern formulation and derives analytic error estimates also for the impact error for homogeneous detector system of many detector layers. The emphasis there was to optimize errors in the low $p_{t}$ regime where multiple scattering is important.

In uniform magnetic field and with negligible multiple scattering the particle trajectory can be modeled as a helix whose projection on the plane normal to the magnetic field is a circular arc. In this work we derive explicit covariance matrix by using the circle description instead of parabola approximation.

This article is organized in the following manner: In section 2 we define the notations and give an introduction to the formalism of the problem. In section 3 we derive the general solution for the covariance matrix independent of the trajectory length or curvature. In section 4 we consider the validity range of the 'straightening hypothesis' which Gluckstern uses in his paper and in section 5 we present simple error formulae applicable in the regime of high $p_{t}$ tracks. Finally in section 6 we consider a few examples and discuss also briefly the treatment of multiple scattering.

\section{Problem formulation}

The coordinate system $(u, v)$ is defined such that the $u$-v-plane is normal to the magnetic field direction. It can be shown that in uniform magnetic field and in the absence of multiple scattering the offset $\varepsilon_{i}$ of a measured point $\left(u_{i}, v_{i}\right)$ from the particle trajectory can be written as [3]:

$$
\varepsilon_{i}=\frac{1}{2} \rho q_{i}^{2}-(1+\rho d)\left(u_{i} \sin \phi-v_{i} \cos \phi\right)+\frac{1}{2} \rho d^{2}+d
$$

where $q_{i}^{2}=u_{i}^{2}+v_{i}^{2}$. The offset $\varepsilon_{i}$ (or residual) is the distance of closest approach (d.c.a.) of a point $\left(u_{i}, v_{i}\right)$ from the trajectory. The three parameters $\rho, \phi$ and $d$ are defined as:

$$
\begin{array}{ll}
\rho= & \text { curvature }(= \pm 1 / R \text { where } R \text { is the radius of curvature }) \\
\phi= & \text { direction of propagation at the point of closest approach } \\
d= & \text { impact parameter }=\text { distance of closest approach to the origin. }
\end{array}
$$

The expression (1) is valid even over a full trajectory loop unlike the parabola approximation used in Refs. [1] and [2]. It is also to be noted that the expression (1) is valid at the straight line limit $\rho \rightarrow 0$. In the absence of point to point correlations the $\chi^{2}$ function for the best fit parameters of the trajectory with $N$ measured points $\left(u_{i}, v_{i}\right)$ reads as:

$$
\chi^{2}=\sum_{i=1}^{N} w_{i} \varepsilon_{i}^{2}
$$

where $w_{i}$ are weights defined as $w_{i}=\sigma_{i}^{-2}$ and $\sigma_{i}$ is the precision of the point $\left(u_{i}, v_{i}\right)$ in the direction normal to the trajectory.

A highly precise explicit solution of the minimizing problem (2) can be written as [3]:

$$
\left\{\begin{array}{l}
\phi=\frac{1}{2} \arctan (2 Q / P) \\
\rho=2\left(\sin \phi \sigma_{u q^{2}}-\cos \phi \sigma_{v q^{2}}\right) / \sigma_{q^{2} q^{2}} \\
d=-\frac{1}{2} \rho\left\langle q^{2}\right\rangle+\langle u\rangle \sin \phi-\langle v\rangle \cos \phi
\end{array}\right.
$$


where $Q=\sigma_{q^{2} q^{2}} \sigma_{u v}-\sigma_{u q^{2}} \sigma_{v q^{2}}$ and $P=\sigma_{q^{2} q^{2}}\left(\sigma_{u u}-\sigma_{v v}\right)-\sigma_{u q^{2}}^{2}+\sigma_{u q^{2}}^{2}$. The coefficients $\sigma_{u u}, \sigma_{u v}, \ldots, \sigma_{q^{2} q^{2}}$ are the six statistical covariances of the measurements $u_{i}, v_{i}$ and $q_{i}^{2}$. The covariance between any two quantities, say $a$ and $b$, is defined in the standard manner as $\sigma_{a b}=\langle a b\rangle-\langle a\rangle\langle b\rangle$ where $\langle a\rangle$ denotes a weighted average e.g. $\langle u\rangle=\sum w_{i} u_{i} / \sum w_{i}$.

The emphasis of this paper is to consider the precision of the three parameters $\rho, \phi$ and $d$ in explicit terms. The covariance matrix of these parameters is calculated as the inverse of the symmetric weight matrix $W$ whose elements are:

$$
W_{k l}=\sum_{i} w_{i} \frac{\partial \varepsilon_{i}}{\partial \alpha_{k}} \frac{\partial \varepsilon_{i}}{\partial \alpha_{l}}
$$

where we have denoted $\rho=\alpha_{1}, \phi=\alpha_{2}$ and $d=\alpha_{3}$. In an arbitrary frame of reference the explicit calculation of (4) and its inverse is infeasible. Using the covariance matrix invariance properties, however, one can invert the $W$ matrix in a straightforward manner as shown in the next section.

\section{Explicit covariance matrix}

When one rotates the coordinate system around the origin by an angle $\phi_{0}$ and shifts the origin by $d_{0}$ in the direction normal to the trajectory, the parameters $\rho, \phi$ and $d$ transform as: $\rho \rightarrow \rho, \phi \rightarrow \phi-\phi_{0}$ and $d \rightarrow d-d_{0}$. It follows that the covariance matrix is invariant under this kind of transformation. The trick to make the analytic inversion of the weight matrix (4) feasible is based on this invariance. Before the inversion the coordinate system is rotated and translated such that:

$$
d=\phi=0
$$

i.e. the new $x$-axis is tangential to the trajectory at the origin (see Fig. 1). We denote the new coordinate axes as $x$ and $y$.

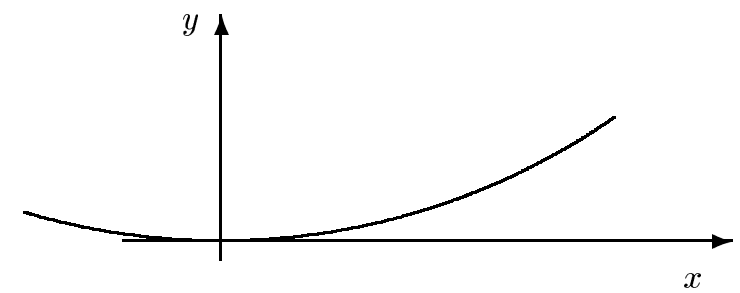

The transformation from $\left(u_{i}, v_{i}\right)$ to $\left(x_{i}, y_{i}\right)$ goes as:

$$
\left\{\begin{array}{l}
x_{i}=+u_{i} \cos \phi+v_{i} \sin \phi \\
y_{i}=-u_{i} \sin \phi+v_{i} \cos \phi-d
\end{array}\right.
$$

where $\phi$ and $d$ are computed from (3).

\subsection{General case}

In the coordinate system of Fig. 1 the three derivatives needed in (4) are simply:

$$
\frac{\partial \varepsilon_{i}}{\partial \rho}=\frac{1}{2} r_{i}^{2}, \quad \frac{\partial \varepsilon_{i}}{\partial \phi}=-x_{i}, \quad \frac{\partial \varepsilon_{i}}{\partial d}=1+\rho y_{i}
$$

with $r_{i}^{2}=x_{i}^{2}+y_{i}^{2}$. The algebra to calculate the weight matrix and its inverse can be found in Appendix 1. As the result we get the covariance matrix elements as functions of statistical moments of the measured 
coordinates:

$$
\left\{\begin{aligned}
\sigma_{\rho \rho} & =C\left[4 \sigma_{x x}-4 \rho^{2}\left(\left\langle x^{2}\right\rangle^{2}-\langle x\rangle\left\langle x r^{2}\right\rangle+\frac{1}{4} \rho^{2}\left\langle x r^{2}\right\rangle^{2}\right)\right] \\
\sigma_{\rho \phi} & =C\left[2 \sigma_{x r^{2}}-\rho^{2}\left(2\left\langle x^{2}\right\rangle\left\langle x r^{2}\right\rangle-\left\langle r^{2}\right\rangle\left\langle x r^{2}\right\rangle-\langle x\rangle\left\langle r^{4}\right\rangle+\frac{1}{2} \rho^{2}\left\langle r^{4}\right\rangle\left\langle x r^{2}\right\rangle\right)\right] \\
\sigma_{\phi \phi} & =C\left[\sigma_{r^{2}} r^{2}+\rho^{2}\left\langle r^{4}\right\rangle\left(\left\langle y^{2}\right\rangle-\frac{1}{4} \rho^{2}\left\langle r^{4}\right\rangle\right)\right] \\
\sigma_{\rho d} & =C\left[2\langle x\rangle\left\langle x r^{2}\right\rangle-2\left\langle x^{2}\right\rangle\left\langle r^{2}\right\rangle-\rho^{2}\left(\left\langle x r^{2}\right\rangle^{2}-\left\langle x^{2}\right\rangle\left\langle r^{4}\right\rangle\right)\right] \\
\sigma_{\phi d} & =C\left[\langle x\rangle\left\langle r^{4}\right\rangle-\left\langle r^{2}\right\rangle\left\langle x r^{2}\right\rangle\right] \\
\sigma_{d d} & =C\left[\left\langle x^{2}\right\rangle\left\langle r^{4}\right\rangle-\left\langle x r^{2}\right\rangle^{2}\right]
\end{aligned}\right.
$$

where $C=\left\{S_{w}\left[\sigma_{x x} \sigma_{r^{2} r^{2}}-\left(\sigma_{x r^{2}}\right)^{2}\right]\right\}^{-1}$ and $S_{w}$ is the sum of weights. The above covariance formulae hold for any curvature and track length even for a closed trajectory loop in the absence of multiple scattering. We have verified these analytic formulae by comparison with a code [4] which performs numerically the calculation and inversion of the weight matrix (4) and found no significant difference.

The error matrix (7) depends on the choice of origin. We call the origin as the reference point of the error matrix. The propagation of errors to a new reference point on the trajectory is simply performed by transforming the coordinate system such that the new $x$-axis is tangential to the trajectory at the new reference point and using the transformed coordinates in eqs. (7). Notice, however, that the curvature variance $\sigma_{\rho \rho}$ is independent of the reference point.

\subsection{Fit with vertex constraint}

In case the emission point (vertex) of the particle is known to certain precision one simply adds it as an extra measured point with appropriate weight and uses the formulation derived above.

In the special case that the trajectory is forced to go through the vertex the formulation becomes somewhat simpler: the impact parameter $d$ vanishes at the vertex and the covariance matrix becomes a $2 \times 2$ matrix. Its derivation is straightforward and we give only the result in the following. We transform the origin of the coordinate system to the vertex point and perform a rotation such that the track direction coincides with the $x$-axis (as in Fig. 1). In this coordinate system the covariance matrix of $\rho$ and $\phi$ becomes:

$$
\left\{\begin{array}{l}
\sigma_{\rho \rho}=C^{\prime} 4\left\langle x^{2}\right\rangle \\
\sigma_{\rho \phi}=C^{\prime} 2\left\langle x r^{2}\right\rangle \\
\sigma_{\phi \phi}=C^{\prime}\left\langle r^{4}\right\rangle
\end{array}\right.
$$

with $C^{\prime}=\left\{S_{w}\left[\left\langle x^{2}\right\rangle\left\langle r^{4}\right\rangle-\left\langle x r^{2}\right\rangle^{2}\right]\right\}^{-1}$. The covariance matrix (8) is defined with the vertex as the reference point.

\subsection{Tracks in zero magnetic field}

In case of zero magnetic field one fits a straight line and the equation of residuals is:

$$
\varepsilon_{i}=-u_{i} \sin \phi+v_{i} \cos \phi+d
$$

Noticing again that the covariance matrix is invariant in rotation and translation normal to the trajectory, we compute the covariance matrix in the system where $\phi=d=0$. A brief calculation gives:

$$
\left\{\begin{array}{l}
\sigma_{\phi \phi}=\left(S_{w} \sigma_{x x}\right)^{-1} \\
\sigma_{\phi d}=\left(S_{w} \sigma_{x x}\right)^{-1}\langle x\rangle \\
\sigma_{d d}=\left(S_{w} \sigma_{x x}\right)^{-1}\left\langle x^{2}\right\rangle
\end{array}\right.
$$

where $x$ is the coordinate measured along the track. One can see that the $\phi$ variance $\sigma_{\phi \phi}$ is invariant in translation of the origin along the trajectory whereas the impact parameter variance $\sigma_{d d}$ is a function of the position. The error propagation of $\sigma_{d d}$ is again made simply by choosing the new reference point as the origin in the formulae (10). One concludes also that the correlation between $\phi$ and $d$ vanishes at the weighted centre of the trajectory.

From eqs. (10) one can derive an illustrative formula for the impact parameter error $\Delta d=\sqrt{\sigma_{d d}}$ in the straight line case:

$$
\Delta d=S_{w}^{-\frac{1}{2}} \oplus \Delta \phi x_{r}
$$

where $\Delta \phi=\sqrt{\sigma_{\phi \phi}}$ and $x_{r}=x-\langle x\rangle$ is the distance of the reference point from the weighted centre of track. This means that the impact parameter error is a quadratic sum of the impact error at the centre of track $\left(=1 / \sqrt{S_{w}}\right)$ and the product of direction error times the distance from the centre. 


\section{Straightening hypothesis}

Gluckstern [1] makes the assumption that the errors are independent of curvature and derives the 2 by 2 error matrix elements $\sigma_{\rho \rho}, \sigma_{\rho \phi}$ and $\sigma_{\phi \phi}$ by fitting parabola to a sample of hits assumed to be measured in zero magnetic field. Gluckstern states that this 'straightening hypothesis' can be proved, but he bypasses the proof. In the following we show that this hypothesis is an approximation valid at the limit of small $\rho L$ where $L$ is the track length in $x y$.

\subsection{Counter examples}

In order to show that Gluckstern's hypothesis is inexact at large curvature we compute $\sigma_{\rho \rho}$ of eq. (7) in special cases and compare the result with the one obtained with a straightened track. Let us take a half circle going through the points $(-1,1),(0,0)$ and $(1,1)$ for which $\rho=1$. When straightened, the corresponding points become $\left(-\frac{1}{2} \pi, 0\right),(0,0)$ and $\left(\frac{1}{2} \pi, 0\right)$. Assuming equal precision $\varepsilon$ (normal to the trajectory) for all points we get:

$$
\sigma_{\rho \rho}(\text { exact })=\frac{\varepsilon^{2}}{2} ; \quad \sigma_{\rho \rho}(\text { Gluckstern })=\frac{96 \varepsilon^{2}}{\pi^{4}} \simeq 0.99 \varepsilon^{2}
$$

which implies about factor 2 difference. When adding $(0,2)$ as a fourth point, the trajectory makes three quarters of a full circle and we get:

$$
\sigma_{\rho \rho}(\text { exact })=\frac{\varepsilon^{2}}{4} ; \quad \sigma_{\rho \rho}(\text { Gluckstern })=\frac{81 \varepsilon^{2}}{(1.5 \pi)^{4}} \simeq 0.16 \varepsilon^{2} .
$$

In the following section we take a closer look and find the validity range of the straightening hypothesis.

\subsection{Validity range}

In order to simplify the calculation we study the validity range of Gluckstern straightening hypothesis in the special case in which: 1) points are uniformly spaced, 2) equally precise and 3) the number of points $N$ is large. We calculate the curvature variance $\sigma_{\rho \rho}$ in (7) as a function of the track length and compare the result with the Gluckstern formula.

Since $\sigma_{\rho \rho}$ is invariant under translation of origin along the trajectory, we place the origin at the centre of track. It follows then from the assumptions 1) and 2) that the terms $\langle x\rangle$ and $\left\langle x r^{2}\right\rangle$ vanish so that $\sigma_{\rho \rho}$ in (7) becomes:

$$
\sigma_{\rho \rho}=\frac{4}{N} \varepsilon^{2} \frac{1-\rho^{2}\left\langle x^{2}\right\rangle}{\left\langle r^{4}\right\rangle-\left\langle r^{2}\right\rangle^{2}}
$$

To calculate the mean values in (12) we use the assumption 3 ) and replace necessary summations by integration. Using $x=2 R \sin \varphi \cos \varphi$ and $r=2 R \sin \varphi$ which are valid when the trajectory is tangential to the $x$-axis at the origin (see Fig. 1), we can perform the necessary integrations along the trajectory as a function of the azimuth angle $\varphi$. The $\varphi$ variable translates to the path length variable $s$ through $\varphi=-\frac{1}{2} \rho / s$ so that the result is a function of the track length $L$.

The calculation is fairly lengthy and the answer is a somewhat complicated trigonometric function for which we have have derived a series expansion including terms up to the second order in $\rho L$ :

$$
\sigma_{\rho \rho}=\frac{720}{N} \frac{\varepsilon^{2}}{L^{4}}\left[1-\frac{1}{21}(\rho L)^{2}+\ldots\right] .
$$

Similar expansions can be obtained for the other elements of the covariance matrix.

In the limit $|\rho L| \rightarrow 0$ the equation (13) approaches the Gluckstern [1] formula derived under the same conditions. One concludes that with the straightening hypothesis the curvature variance gets overestimated by a factor of about $|\rho L|^{2} / 21$ which is less than $2 \%$ for $|\rho L|<0.65$. This is equivalent to saying that the estimate of $\Delta p_{t} / p_{t}$ by straightening method is in error by less than $1 \%$ for tracks with total turning angle less than about 40 degrees.

Usually one is interested in the detector performance at the high $p_{t}$ limit. The above condition implies $p_{t}[\mathrm{GeV}]=0.3 B L /|\rho L|>0.46 B[\mathrm{~T}] L[\mathrm{~m}]$. For as high as a 4 Tesla field and $1 \mathrm{~m}$ projected track length the limit is as low as $p_{t}>1.8 \mathrm{GeV}$. 


\section{Practical error formulae}

In the previous section we showed that for practical purposes one gets precise error formulae by performing the calculation in the trajectory system, i.e. as a function of length along the track, and neglecting terms higher or equal to second order in $\rho L$. The elements of the curvature, direction and impact parameter covariance matrix become then:

$$
\left\{\begin{array}{rlrl}
\sigma_{\rho \rho} & =4 D\left[\left\langle s^{2}\right\rangle-\langle s\rangle^{2}\right] & & \left(=4 D \sigma_{s s}\right) \\
\sigma_{\rho \phi} & =2 D\left[\left\langle s^{3}\right\rangle-\langle s\rangle\left\langle s^{2}\right\rangle\right] & & \left(=2 D \sigma_{s s^{2}}\right) \\
\sigma_{\phi \phi} & =D\left[\left\langle s^{4}\right\rangle-\left\langle s^{2}\right\rangle^{2}\right] & & \left(=D \sigma_{s^{2}} s^{2}\right) \\
\sigma_{\rho d} & =2 D\left[\langle s\rangle\left\langle s^{3}\right\rangle-\left\langle s^{2}\right\rangle^{2}\right] & \\
\sigma_{\phi d}= & D\left[\langle s\rangle\left\langle s^{4}\right\rangle-\left\langle s^{2}\right\rangle\left\langle s^{3}\right\rangle\right] & \\
\sigma_{d d} & =D\left[\left\langle s^{2}\right\rangle\left\langle s^{4}\right\rangle-\left\langle s^{3}\right\rangle^{2}\right] &
\end{array}\right.
$$

where $D=\left\{S_{w}\left[\sigma_{s s} \sigma_{s^{2} s^{2}}-\left(\sigma_{s s^{2}}\right)^{2}\right]\right\}^{-1}$ and $s$ denotes the path length coordinate. With these formulae the particle measurement precisions can be calculated with just a few lines of program code. They are valid for any spacing of points and variable detector resolutions. The formulae are in error by less than $1 \%$ for trajectory turning angle $L / R$ up to about 0.65 radians.

For small curvatures one can further simplify (14) by replacing the path length $s$ by the radial coordinate: $s_{i} \leftarrow r_{i}$. The precision suffers a little so that the $1 \%$ accuracy for $\Delta \rho=\sqrt{\sigma_{\rho \rho}}$ is valid for $L / R<0.3$ i.e. for $p_{t}>4 \mathrm{GeV}$, if $B=4$ Tesla and $L=1 \mathrm{~m}$.

In the following we consider a few special cases and derive simple expressions for the covariance matrix elements.

\subsection{Uniform spacing of points}

With equal spacing $\Delta s=L /(N-1)$ and equal weights of points we can derive even simpler expressions for the covariances (14). We have $s_{i}=s_{0}+(i-1) \Delta s$. Hence the mean values in (14) involve computation of the four series $S_{N}(k)=\sum_{i=1}^{N} i^{k}$ where $k$ takes values from 1 to 4 . The calculation is fairly straightforward algebra and we give only the result in the following:

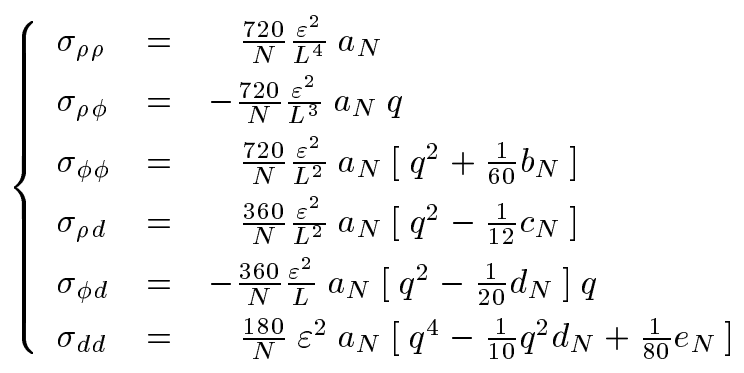

where $\varepsilon$ is the detector resolution and the 'propagation parameter' $q$ is defined as $q=(s-\langle s\rangle) / L$. The constants

$$
a_{N}=\frac{(N-1)^{3}}{\left(N^{2}-4\right)(N+1)}, b_{N}=\frac{N^{2}-4}{(N-1)^{2}}, c_{N}=\frac{N+1}{N-1}, d_{N}=\frac{N^{2}+1}{(N-1)^{2}}, e_{N}=c_{N} \frac{3 N^{2}-7}{3(N-1)^{2}}
$$

all approach to unity in the limit of large number of points $N$. Gluckstern [1] has derived equivalent formulae for $\sigma_{\rho \rho}, \sigma_{\rho \phi}$ and $\sigma_{\phi \phi}$ at the beginning of the track $(q=-0.5)$.

For comparison we give also the 2 by 2 covariance matrix for the straight line fit $(B=0)$ with equal spacing and precision of points:

$$
\sigma_{\phi \phi}=\frac{12}{N} \frac{\varepsilon^{2}}{L^{2}} f_{N}, \quad \sigma_{\phi d}=\frac{12}{N} \frac{\varepsilon^{2}}{L} q f_{N}, \quad \sigma_{d d}=\frac{1}{N} \varepsilon^{2}\left(1+12 q^{2} f_{N}\right)
$$

where $f_{N}=(N-1) /(N+1)$. Notice that the variance of the impact parameter increases quadratically as a function of the extrapolation length $q$. This is to be compared with the case of non-zero magnetic field where the increase goes as fourth power in $q$. 


\subsection{Best curvature precision}

Suppose we have $N$ detectors with equally good point resolution $\varepsilon$. Then one may ask what kind of detector spacing gives the best momentum resolution. In the following we consider the problem for both unconstrained and vertex constrained fits.

\section{Unconstrained fit}

We calculate $\sigma_{\rho \rho}$ in the frame of reference where $\langle s\rangle=0$ so that according to (14) we have

$$
\sigma_{\rho \rho}=\frac{4\left\langle s^{2}\right\rangle}{S_{w}\left[\left\langle s^{2}\right\rangle \sigma_{s^{2}} s^{2}-\left\langle s^{3}\right\rangle^{2}\right]} .
$$

It turns out that for minimal $\sigma_{\rho \rho}$ the points must be placed symmetrically about $\langle s\rangle=0$ so that $\left\langle s^{3}\right\rangle$ vanishes. Then the curvature variance is:

$$
\sigma_{\rho \rho}=\frac{4}{S_{w}\left(\left\langle s^{4}\right\rangle-\left\langle s^{2}\right\rangle^{2}\right)}
$$

Minimizing (18) with respect to all $s_{i}$ with $\left|s_{i}\right|<0.5 L$ (inner points) implies $s_{i}\left(s_{i}{ }^{2}-\left\langle s_{i n}\right\rangle^{2}\right)=0$. This is possible only if $s_{i}=0, i=1, \ldots, M$, i.e. all $M<N$ inner points must be placed at the centre of track. Inserting this result into (18) we find that the best curvature precision is:

$$
\sigma_{\rho \rho}=\frac{64 \varepsilon^{2}}{L^{4}} \frac{N}{M(N-M)} .
$$

To optimize (19) with respect to the number of inner points one has to determine $M$ such that the product $M(N-M)$ becomes maximal for fixed $N$. For example, if the total number of points $N$ is divisible by 4 , the smallest possible curvature variance is obtained when $N / 2$ measurements are at the centre of track and $N / 4$ at both ends. Then the curvature variance is:

$$
\sigma_{\rho \rho}=\frac{256 \varepsilon^{2}}{N L^{4}} .
$$

This result was obtained by Gluckstern [1] with a different approach. Notice, however, that the formula (19) should be used, if $N$ is not a multiple of 4 . For instance if $N=11$, the best distribution of the number of measurements is 3-5-3 at the beginning-centre-end positions.

\section{Vertex constrained fit}

In this case we calculate $\sigma_{\rho \rho}$ with the origin as the reference point. Using the path length coordinate $s$ we get from eq. (8):

$$
\sigma_{\rho \rho}=\frac{4\left\langle s^{2}\right\rangle}{S_{w}\left[\left\langle s^{2}\right\rangle\left\langle s^{4}\right\rangle-\left\langle s^{3}\right\rangle^{2}\right]} .
$$

Minimizing this with respect to the inner points $0<s_{i}<L$ we find that

$$
s_{i}=s_{0}=0.5\left\langle s^{3}\right\rangle /\left\langle s^{2}\right\rangle
$$

which implies that for the best momentum precision the inner measurements should be concentrated at the same position: $s_{i}=\beta L, i=1, \ldots, M$ with $0<\beta<1$ and $M<N$. Inserting $s_{0}=\beta L$ in (22) we derive the following 3rd order equation for the optimal position $\beta$ for any given $M$ :

$$
\frac{M}{N-M} \beta^{3}+2 \beta-1=0 .
$$

One can find the optimal fraction $P=M / N$ by considering $P$ as a continuous variable (large $N$ ) and minimizing (21) with respect to it. A brief calculation gives:

$$
P=\frac{1}{\sqrt{2}} \quad \text { and } \quad \beta=\sqrt{2}-1
$$


giving the best curvature variance of

$$
\sigma_{\rho \rho}=(68+48 \sqrt{2}) \frac{\varepsilon^{2}}{N L^{4}} \simeq \frac{136 \varepsilon^{2}}{N L^{4}}
$$

This means that the best transverse momentum precision in case of vertex constrained fit is obtained by concentrating about $71 \%$ of the measurements near the position $s_{0}=0.41 L$ and the remaining $29 \%$ at the end of the trajectory.

\section{Discussion}

\section{Examples}

In the following we illustrate the usage of the formulae derived above with a couple of examples. The transverse momentum is a function of curvature and magnetic field: $p_{t}[\mathrm{GeV}]=0.3 B / \rho[\mathrm{T}][\mathrm{m}]$. It follows that the relative error on transverse momentum reads:

$$
\frac{\Delta p_{t}}{p_{t}}=\frac{\Delta \rho}{0.3 B} p_{t}
$$

where $\Delta \rho$ depends on the detector resolutions and spacing. Utilizing eq. (14) and taking the high $p_{t}$ limit so that we can use the radial coordinates $r$ instead of $s$ we get:

$$
\frac{\Delta p_{t}}{p_{t}}=\frac{\left[S_{w}\left(\sigma_{r^{2} r^{2}}-\left(\sigma_{r r^{2}}\right)^{2} / \sigma_{r r}\right)\right]^{-\frac{1}{2}}}{0.3 B} p_{t}
$$

Let us assume that there are 5 points measured with $20 \mu \mathrm{m}$ precision and another 6 points measured with $50 \mu \mathrm{m}$ precision in a $4 \mathrm{~T}$ field. We assume further that the first point is at $0.05 \mathrm{~m}$ from the origin and that the point spacing is constant $0.1 \mathrm{~m}$. The resulting precisions are then:

$$
\frac{\Delta p_{t}}{p_{t}}=17 \% p_{t}\left[\mathrm{TeV}^{-1}\right], \quad \Delta \phi=0.1 \mathrm{mrad}, \quad \Delta d=20 \mu \mathrm{m}
$$

where $\Delta \phi$ and $\Delta d$ are also calculated from (14). Adding the vertex point and assuming $10 \mu \mathrm{m}$ precision gives: $\Delta p_{t} / p_{t}=13 \% p_{t}\left[\mathrm{TeV}^{-1}\right], \Delta \phi=60 \mu \mathrm{rad}$ and $\Delta d=9 \mu \mathrm{m}$.

\section{Full covariance matrix}

So far we have considered the projection of the particle trajectory on the bending plane. The trajectory in 3D space is described by 5 parameters for which we choose $\rho, \phi$ and $d$ and the following two parameters:

$$
\begin{aligned}
& \lambda=\quad \text { angle between the trajectory and the } x y \text { plane ('dip' angle) } \\
& z_{0}=\quad z \text {-coordinate of the d.c.a. point. }
\end{aligned}
$$

With this parametrization the 3D coordinates of the d.c.a. point on the trajectory are $\left(d \sin \phi,-d \cos \phi, z_{0}\right)$. The analytic calculation of the full 5 by 5 covariance matrix is not quite feasible. Instead, it is a straightforward calculation to derive the five parameters covariance matrix as a composition of diagonal blocks of a 3 by 3 matrix (for $\rho, \phi, d$ ) derived above and a 2 by 2 matrix (for $\lambda, z_{0}$ ). In this representation one ignores the possible correlation between the $x y$ and $z$ measurements, but the result is normally a very good approximation. For completeness we give the explicit covariance terms of the fitted $\lambda$ and $z_{0}$ in the limit of large $p_{t}$ :

$$
\left\{\begin{array}{lll}
\sigma_{\lambda \lambda} & =\left(S_{v} \sigma_{r r}\right)^{-1}\left[1+\left(\sigma_{z r} / \sigma_{r r}\right)^{2}\right]^{-2} \\
\sigma_{\lambda z_{0}} & = & -\left(S_{v} \sigma_{r r}\right)^{-1}\left[1+\left(\sigma_{z r} / \sigma_{r r}\right)^{2}\right]^{-1}\langle r\rangle \\
\sigma_{z_{0} z_{0}} & = & \left(S_{v} \sigma_{r r}\right)^{-1}\left\langle r^{2}\right\rangle
\end{array}\right.
$$

where $S_{v}$ is the sum of $z$ weights: $S_{v}=\sum \Delta z_{i}{ }^{-2}$ and $r$ is the radial coordinate as before. For fitted $\lambda$ and $z_{0}$ we have $\tan \lambda=\sigma_{z r} / \sigma_{r r}$ and $z_{0}=\langle z\rangle-\langle r\rangle \tan \lambda$. 


\section{Multiple scattering}

To a good approximation the multiple scattering (m.s.) contribution to the trajectory errors can be added quadratically to the errors due to detector resolution to obtain the total error. A more precise treatment of the m.s. contribution should be done numerically using e.g. the method described in Ref. [5].

Here we just take an example for curvature variation due to m.s. which is given by [6]:

$$
\Delta \rho(\text { m.s. }) \simeq \frac{0.016[\mathrm{GeV}]}{p_{t}} \frac{1}{L_{s} \cos \lambda} \sqrt{\frac{L_{s}}{L_{0}}}
$$

where $L_{0}$ is the radiation length and $L_{s}$ is the trajectory length in space. Taking the above simple example and assuming that the detector 'thickness' in units of radiation lengths is $L_{s} / L_{0}=20 \%$ at $\lambda=0$ we get:

$$
\frac{\Delta p_{t}}{p_{t}} \simeq 0.006 \oplus 0.17 p_{t}\left[\mathrm{TeV}^{-1}\right]
$$

We see that in this case multiple scattering becomes significant for $p_{t}<30 \mathrm{GeV}$ and the relative transverse momentum error levels off to a constant value at lower transverse momenta.

\section{Summary}

We have derived the explicit covariance matrix (7) of particle trajectory curvature, azimuth angle and impact parameter valid for full range of curvatures at the limit of negligible multiple scattering. The formulae are valid for hybrid detector systems with variable measurement precisions. We consider a number of interesting special cases: homogeneous detector system, inclusion of the vertex constraint and the case of zero magnetic field. We also consider the Gluckstern 'straightening hypothesis' and show that it is an approximation good for small curvatures. We derive simplified covariance formulae (14) valid for high transverse momenta and discuss some practical examples. We also discuss briefly the effect of multiple scattering.

\section{References}

[1] R.L. Gluckstern, Nucl. Instr. and Meth. 24 (1963) 352.

[2] W.R. Innes, Nucl. Instr. and Meth. A329 (1993) 238.

[3] V. Karimäki, Nucl. Instr. and Meth. A305 (1991) 187.

[4] V. Karimäki, Comp. Phys. Commun. 69 (1992) 133.

[5] P. Billoir, Nucl. Instr. and Meth. 225 (1984) 352.

[6] Particle Data Group, Phys. Review D54 (1996) 1. 


\section{Appendix I: Inversion of the weight matrix}

In the following we show the main steps in the calculation of the covariance matrix elements (7). With derivatives (6) the weight matrix (4) becomes:

$$
W=S_{w}\left(\begin{array}{ccc}
\frac{1}{4}\left\langle r^{4}\right\rangle & -\frac{1}{2}\left\langle x r^{2}\right\rangle & \frac{1}{2}\left\langle r^{2}\right\rangle+\frac{1}{2} \rho\left\langle y r^{2}\right\rangle \\
-\frac{1}{2}\left\langle x r^{2}\right\rangle & \left\langle x^{2}\right\rangle & -\langle x\rangle-\rho\langle x y\rangle \\
\frac{1}{2}\left\langle r^{2}\right\rangle+\frac{1}{2} \rho\left\langle y r^{2}\right\rangle & -\langle x\rangle-\rho\langle x y\rangle & 1+2 \rho\langle y\rangle+\rho^{2}\left\langle y^{2}\right\rangle
\end{array}\right)
$$

where we use the notation $S_{w}=\sum w_{i}$. Using the identities

$$
\frac{1}{2} \rho\left\langle r^{2}\right\rangle=-\langle y\rangle, \quad \frac{1}{2} \rho\left\langle r^{4}\right\rangle=-\left\langle y r^{2}\right\rangle, \quad \frac{1}{2}\left\langle x r^{2}\right\rangle=-\langle x y\rangle
$$

which follow from (3) and (5), the matrix (A.1) transforms to:

$$
W=S_{w}\left(\begin{array}{ccc}
\frac{1}{4}\left\langle r^{4}\right\rangle & -\frac{1}{2}\left\langle x r^{2}\right\rangle & \frac{1}{2}\left\langle r^{2}\right\rangle-\frac{1}{4} \rho^{2}\left\langle r^{4}\right\rangle \\
-\frac{1}{2}\left\langle x r^{2}\right\rangle & \left\langle x^{2}\right\rangle & -\langle x\rangle+\frac{1}{2} \rho^{2}\left\langle x r^{2}\right\rangle \\
\frac{1}{2}\left\langle r^{2}\right\rangle-\frac{1}{4} \rho^{2}\left\langle r^{4}\right\rangle & -\langle x\rangle+\frac{1}{2} \rho^{2}\left\langle x r^{2}\right\rangle & 1-\rho^{2}\left\langle x^{2}\right\rangle
\end{array}\right)
$$

Notice that equations (3) are valid for the transformed coordinates with solutions (5).

In order to invert (A.3) we have to calculate det $W$. After a brief manipulation of the rows and columns the determinant becomes:

$$
\operatorname{det} W=S_{w}{ }^{3}\left|\begin{array}{ccc}
\frac{1}{4}\left\langle r^{4}\right\rangle & -\frac{1}{2}\left\langle x r^{2}\right\rangle & \frac{1}{2}\left\langle r^{2}\right\rangle \\
-\frac{1}{2}\left\langle x r^{2}\right\rangle & \left\langle x^{2}\right\rangle & -\langle x\rangle \\
\frac{1}{2}\left\langle r^{2}\right\rangle & -\langle x\rangle & 1+\Delta
\end{array}\right|
$$

with $\Delta=\rho^{2}\left\langle y^{2}\right\rangle-\frac{1}{2} \rho^{4}\left\langle r^{4}\right\rangle \simeq(\rho \bar{\varepsilon})^{2}$ so that $\Delta$ is highly negligible and we can set $\Delta \simeq 0(\bar{\varepsilon}$ is the average point measurement error). Then with little further manipulation of rows and columns we get a remarkably simple expression for the determinant:

$$
\operatorname{det} W=S_{w}{ }^{3}\left|\begin{array}{ccc}
\frac{1}{4} \sigma_{r^{2} r^{2}} & -\frac{1}{2} \sigma_{x r^{2}} & 0 \\
-\frac{1}{2} \sigma_{x r^{2}} & \sigma_{x x} & 0 \\
0 & 0 & 1
\end{array}\right|=\frac{1}{4} S_{w}{ }^{3}\left[\sigma_{x x} \sigma_{r^{2} r^{2}}-\left(\sigma_{x r^{2}}\right)^{2}\right]
$$

The explicit expressions (7) of the covariance matrix elements are then readily derived from (A.3) and (A.5). 